\title{
TRANSCENDENTALITY OF ZEROS OF HIGHER DERIVATIVES OF FUNCTIONS INVOLVING BESSEL FUNCTIONS
}

\section{LEE LORCH and MARTIN E. MULDOON}

Department of Mathematics and Statistics

York University

North York, Ontario

Canada M3J 1P3

(Received September 2, 1995 and in revised form February 2, 1994)

ABSTRACT. C.L. Siegel established in 1929 [Ges. Abh., v.1, pp. 209-266] the deep results that (i) all zeros of $J_{\nu}(x)$ and $J_{\nu}^{\prime}(x)$ are transcendental when $\nu$ is rational, $x \neq 0$, and (ii) $J_{\nu}^{\prime}(x) / J_{\nu}(x)$ is transcendental when $\nu$ is rational and $x$ algebraic. As usual, $J_{\nu}(x)$ is the Bessel function of first kind and order $\nu$. Here it is shown that simple arguments permit one to infer from Siegel's results analogous but not identical properties of the zeros of higher derivatives of $x^{-\mu} J_{\nu}(x)$ when $\mu$ is algebraic and $\nu$ rational. In particular, $J_{1}^{\prime \prime \prime}( \pm \sqrt{3})=0$ while all other zeros of $J_{1}^{\prime \prime \prime}(x)$ and all zeros of $J_{\nu}^{\prime \prime \prime}(x), \nu^{2} \neq 1, x \neq 0$, are transcendental. Further, $J_{0}^{(4)}( \pm \sqrt{3})=0 \quad$ while all other zeros of $J_{0}^{(4)}(x), \quad x \neq 0$, and of $J_{\nu}^{(4)}(x), \quad \nu \neq 0, x \neq 0$, are transcendental. All zeros of $J_{\nu}^{(n)}(x), \quad x \neq 0$, are transcendental, $n=5, \ldots, 18$, when $\nu$ is rational. For most values of $n$, the proofs used the symbolic computation package Maple $\mathrm{V}$ (Release 1).

KEY WORDS AND PHRASES. Bessel functions, zeros, transcendentality, differential equations.

1991 AMS SUBJECT CLASSIFICATION CODES. 33A40, 11J81

\section{INTRODUCTION.}

Carl Ludwig Siegel established [3;2, Ch. 6, $§ 2$, p. 217] the deep results that when $\nu$ is rational the zeros of $J_{\nu}(x), J_{\nu}^{\prime}(x), x \neq 0$, are transcendental and that the ratio $J_{\nu}^{\prime}(x) / J_{\nu}(x)$ is transcendental for $x$ algebraic. Here $J_{\nu}(x)$ is, as usual, the Bessel function of the first kind and order $\nu$.

From these properties, as will be shown below, analogous (but not always identical) results can be inferred by quite simple arguments for the zeros of higher derivatives of

$$
\mathcal{J}_{\nu, \mu}(x)=x^{-\mu} J_{\nu}(x)
$$

when $\mu$ is algebraic and $\nu$ rational.

For $\mu=0$, this gives full information on the transcendentality of zeros of $J_{\nu}^{(n)}(x), n=$ $2, \ldots, 18$. Other standard special cases are $\mu=\frac{1}{2}, \quad \nu=m+\frac{1}{2}, \quad m=0, \pm 1, \pm 2, \ldots$, the familiar spherical Bessel functions (a multiplicative constant aside), and $\mu=\nu$ which presents the standard entire function $\Lambda_{\nu}(x)$, again disregarding a multiplicative constant. 
The transition from Siegel's theorem to these results is via minor manipulations of the differential equation for $y=\mathcal{J}_{\nu, \mu}(x)[5, \S 4.31(19)$, p. 98]

$$
x^{2} y^{\prime \prime}+(2 \mu+1) x y^{\prime}+\left(x^{2}-\nu^{2}+\mu^{2}\right) y=0 .
$$

These calculations can be applied equally well to the general form

$$
x^{2} y^{\prime \prime}+p_{2}(x) x y^{\prime}+q_{2}(x) y=0,
$$

where $p_{2}(x)$ and $q_{2}(x)$ are algebraic polynomials.

For $y$ satisfying (2), it follows by induction that $y$ also satisfies, for $n=2,3,4, \ldots$,

$$
x^{n} y^{(n)}+p_{n}(x) x y^{\prime}+q_{n}(x) y=0,
$$

where $p_{n}(x), \quad q_{n}(x)$ are algebraic polynomials. The subscript $n$ does not suggest the degrees of these polynomials. (In fact, $\operatorname{deg}\left(p_{n}\right)=2[(n-1) / 2]$ and $\operatorname{deg}\left(q_{n}\right)=2[n / 2]$.) It records only their association with the differential equation (3) of order $n$.

The induction from order $n$ to order $n+1$ leads to

$$
\begin{aligned}
x^{n+1} y^{(n+1)}+\left[x p_{n}^{\prime}(x)\right. & \left.+p_{n}(x)\left\{1-n-p_{2}(x)\right\}+q_{n}(x)\right] x y^{\prime} \\
& +\left[x q_{n}^{\prime}(x)-n q_{n}(x)-q_{2}(x) p_{n}(x)\right] y=0,
\end{aligned}
$$

where the coefficient functions are again polynomials. The passage is achieved by differentiating (3), multiplying through by $x$, then replacing $x^{n} y^{(n)}$ from (3) and $x^{2} y^{\prime \prime}$ from (2). From (4), we have

$$
\begin{gathered}
p_{n+1}=x p_{n}^{\prime}(x)+p_{n}(x)\left\{1-n-p_{2}(x)\right\}+q_{n}(x), \\
q_{n+1}=x q_{n}^{\prime}(x)-n q_{n}(x)-q_{2}(x) p_{n}(x) .
\end{gathered}
$$

Thus, (3) and (4) express the higher derivatives of $\mathcal{J}_{\nu, \mu}(x)$ in terms of $\mathcal{J}_{\nu, \mu}(x)$ and $\mathcal{J}_{\nu, \mu}^{\prime}(x)$.

This will permit the application of Siegel's theorems to $\mathcal{J}_{\nu, \mu}^{(n)}(x)$, since

$$
\frac{\mathcal{J}_{\nu, \mu}^{\prime}(x)}{\mathcal{J}_{\nu, \mu}(x)}=\frac{J_{\nu}^{\prime}(x)}{J_{\nu}(x)}-\frac{\mu}{x}
$$

Hence, $\mathcal{J}_{\nu, \mu}^{\prime}(x) / \mathcal{J}_{\nu, \mu}(x)$ is transcendental whenever $x(\neq 0)$ and $\mu$ are algebraic and $\nu$ is rational, since this is the case for $J_{\nu}^{\prime}(x) / J_{\nu}(x)$. One observation is immediate from Siegel's work and does not require an appeal to the differential equation:

THEOREM 1 . If $\nu$ is rational and $\mu$ algebraic, then any zero of $\mathcal{J}_{\nu, \mu}^{\prime}(x), x \neq 0$, is transcendental.

PROOF. Let $\xi \neq 0$ be any arbitrary zero of $\mathcal{J}_{\nu, \mu}^{\prime}(x)$. From (7),

$$
\frac{J_{\nu}^{\prime}(\xi)}{J_{\nu}(\xi)}=\frac{\mu}{\xi}
$$

If $\xi$ were algebraic, then this equation would imply that $J_{\nu}^{\prime}(\xi) / J_{\nu}(\xi)$ is algebraic. But [2, Ch.6, $\S 2$, p.217] this is false when $\nu$ is rational as here.

Proofs of the remaining results will use special cases of (3). The next theorem, however, will be phrased more generally so as to make it potentially applicable to yet other cases.

THEOREM 2. Let $y(x)$ be a non-trivial solution of the differential equation (3) where $p_{n}(x), q_{n}(x)$ are algebraic polynomials. Suppose for each algebraic $x \neq 0$, that (i) $y(x) \neq 0$ 
and that (ii) $y^{\prime}(x) / y(x)$ is transcendental. Then a zero $\xi \neq 0$ of $y^{(n)}(x)$ is algebraic if and only if $p_{n}(\xi)=q_{n}(\xi)=0$.

PROOF. It is obvious that $\xi \neq 0$ is an algebraic zero of $y^{(n)}(x)$ if $p_{n}(\xi)=q_{n}(\xi)=0$. What remains, then, is to show that $y^{(n)}(\xi)=0, \quad \xi \neq 0$, implies $p_{n}(\xi)=q_{n}(\xi)=0$ when $\xi$ is algebraic.

With $\xi \neq 0$ algebraic, it is immediate from hypotheses (i) and (ii) that $y^{\prime}(\xi) \neq 0$. From $(3), \xi p_{n}(\xi) y^{\prime}(\xi)+q_{n}(\xi) y(\xi)=0$. If $p_{n}(\xi) \neq 0$, then

$$
\frac{y^{\prime}(\xi)}{y(\xi)}=-\frac{q_{n}(\xi)}{\xi p_{n}(\xi)}
$$

The right hand side is algebraic for $\xi \neq 0$ algebraic but, by hypothesis, the left hand side is not.

Hence $p_{n}(\xi)=0$ and so $q_{n}(\xi) y(\xi)=0$. Furthermore, $q_{n}(\xi)$ also vanishes, according to (i), and the proof is complete.

Thus, the algebraic zeros other than 0 , if any, are the common roots of $p_{n}(x)$ and $q_{n}(x)$. The search for such can be made via various methods of elimination, going back, say, to Euler, Bézout and Sylvester.

An application of Theorem 2 to $\mathcal{J}_{\nu, \mu}(x)$, into which Theorem 1 is incorporated, can be formulated as follows:

COROLLARY. If $\nu$ is rational and $\mu$ algebraic, then all but a finite number (perhaps none) of the infinitely many zeros of $\mathcal{J}_{\nu, \mu}^{(n)}(x)$ are transcendental, $x \neq 0, \quad n=0,1,2, \ldots$

The remaining results use the specific differential equations (1) and (4).

THEOREM 3. If $\nu$ is rational and $\mu$ is algebraic, then any zero $\xi \neq 0$ of $\mathcal{J}_{\nu, \mu}^{\prime \prime}(x)$ is transcendental unless $\mu=-\frac{1}{2}$ and $\nu^{2} \neq \frac{1}{4}$. In the latter cases there are precisely two algebraic zeros, $\pm \xi \neq 0$ where $\xi^{2}=\nu^{2}-\frac{1}{4}$.

REMARK. Note that these algebraic zeros are imaginary when $\nu^{2}<1 / 4$.

COROLLARY. All zeros, other than 0 , of $J_{\nu}^{\prime \prime}(x),\left[J_{\nu}(x) / x^{\frac{1}{2}}\right]^{\prime \prime}$ and $\left[J_{\nu}(x) / x^{\nu}\right]^{\prime \prime}$ are transcendental when $\nu$ is rational.

PROOF. Applying Theorem 2 to differential equation (1) shows that a zero $\xi \neq 0$ of $\mathcal{J}_{\nu, \mu}^{\prime \prime}(x)$ is algebraic when and only when $p_{2}(\xi)=2 \mu+1$ and $q_{2}(\xi)=\xi^{2}-\nu^{2}+\mu^{2}$ vanish simultaneously. This establishes Theorem 3. (It may be noted that $\sqrt{x} J_{\frac{1}{2}}(x)=\sqrt{2 / \pi} \sin x$ and $\sqrt{x} J_{-\frac{1}{2}}(x)=\sqrt{2 / \pi} \cos x$. The zeros, other than 0 , of their respective second derivatives are all non-zero rational multiples of $\pi$ and hence transcendental.) The Corollary is immediate.

For $\mathcal{J}_{\nu, \mu}^{\prime \prime \prime}(x)$, non-zero algebraic zeros arise even when $\mu=0$, i.e., for $J_{\nu}^{\prime \prime \prime}(x)$. Specifically, $J_{\nu}^{\prime \prime \prime}( \pm \sqrt{3})=0$ when $\nu^{2}=1$. All other zeros, of $J_{\nu}^{\prime \prime \prime}(x)$ are transcendental for any rational $\nu$ including \pm 1 .

THEOREM 4. If $\nu$ is rational and $\mu$ algebraic, each zero $\xi \neq 0$ of $\mathcal{J}_{\nu, \mu}^{\prime \prime \prime}(x)$ is transcendental except when $\nu^{2}=\mu^{2}+(\mu+1)(2 \mu+1)^{2}=4 \mu^{3}+9 \mu^{2}+5 \mu+1$, with $\xi^{2}=$ $(\mu+1)(2 \mu+1)(2 \mu+3)$ and with $\left(4 \mu^{3}+9 \mu^{2}+5 \mu+1\right)^{\frac{1}{2}}$ rational.

COROLLARY. For $\nu$ rational, (i) any zero $\xi \neq 0$ of $J_{\nu}^{\prime \prime \prime}(x)$ is transcendental except when $\nu^{2}=1$ with $\xi^{2}=3, \quad$ (ii) any zero $\xi \neq 0$ of $\left[J_{\nu}(x) / x^{\frac{1}{2}}\right]^{\prime \prime \prime}$ is transcendental except when $\nu^{2}=\frac{25}{4}$ with $\xi^{2}=12$, and (iii) all zeros (other than zero) of $\left[J_{\nu}(x) / x^{ \pm \nu}\right]^{\prime \prime \prime}$ and of $\left[J_{\nu}(x) / x^{ \pm 1}\right]^{\prime \prime \prime}$ are transcendental without exception. 
PROOF. When $n=2$ and $y=\mathcal{J}_{\nu, \mu}(x)$, the differential equation (4) becomes

$$
\begin{aligned}
x^{3} y^{\prime \prime \prime}+x\left[x^{2}-\nu^{2}\right. & \left.+\mu^{2}-2(\mu+1)(2 \mu+1)\right] y^{\prime} \\
& -\left[(2 \mu+1) x^{2}+(2 \mu+3)\left(\mu^{2}-\nu^{2}\right)\right] y=0 .
\end{aligned}
$$

The coefficients of $y$ and $x y^{\prime}$ vanish simultaneously when and only when $\nu^{2}=\mu^{2}+(\mu+$ 1) $(2 \mu+1)^{2}$ with $x^{2}=(\mu+1)(2 \mu+1)(2 \mu+3)$, as asserted in the theorem. In addition, since $\nu$ is rational, so too must be $\left[\mu^{2}+(\mu+1)(2 \mu+1)^{2}\right]^{\frac{1}{2}}$.

This establishes the theorem. Parts (i) and (ii) of the corollary are immediate. When $\mu^{2}=\nu^{2}$, the foregoing shows that coefficients of $y$ and $x y^{\prime}$ vanish only when $\mu=-1,-\frac{1}{2}$ so that $x=0$. This verifies the assertion in (iii) when $\mu= \pm \nu$. If $\mu=1$, then $\nu^{2}=19$, so that $\nu$ cannot be rational as required. If $\mu=-1$, then $x^{2}=0$. Together these last two sentences verify the remaining assertion in part (iii) of the corollary.

The well-known relation $J_{1}(x)=-J_{0}^{\prime}(x)$ makes it obvious that $J_{0}^{(4)}( \pm \sqrt{3})=-J_{1}^{\prime \prime \prime}( \pm \sqrt{3})=$ 0 , so that $J_{\nu}^{(4)}(x)$ possesses a pair of algebraic zeros (other than zero) for a specific rational $\nu$, namely $\nu=0$. It will be seen that all other zeros (other than zero) of $J_{\nu}^{(4)}(x)$ are transcendental when $\nu$ is rational, including those for which $\nu=0, \quad x^{2} \neq 3$.

This will emerge from the following theorem.

THEOREM 5. If $\nu$ is rational and $\mu$ algebraic, then any zero $\xi \neq 0$ of $\mathcal{J}_{\nu, \mu}^{(4)}(x)$ is transcendental except when

$$
\begin{aligned}
\nu^{2}= & \mu^{2}-\frac{1}{4}(2 \mu+1)\left[4 \mu^{2}+12 \mu+7\right. \\
& \left. \pm\left(32 \mu^{4}+160 \mu^{3}+284 \mu^{2}+204 \mu+49\right)^{\frac{1}{2}}\right]
\end{aligned}
$$

with

$$
\xi^{2}=-\frac{1}{4}(2 \mu+3)\left[-4 \mu^{2}-12 \mu-7 \pm\left(32 \mu^{4}+160 \mu^{3}+284 \mu^{2}+204 \mu+49\right)^{\frac{1}{2}}\right]
$$

and with

$$
\left\{\mu^{2}-\frac{1}{4}(2 \mu+1)\left[\left(4 \mu^{2}+12 \mu+7\right) \pm\left(32 \mu^{4}+160 \mu^{3}+284 \mu^{2}+204 \mu+49\right)^{\frac{1}{2}}\right]\right\}^{\frac{1}{2}}
$$

rational.

COROLLARY. For $\nu$ rational, (i) each zero $\xi \neq 0$ of $J_{\nu}^{(4)}(x)$ is transcendental except when $\nu=0$ with $\xi^{2}=3$, (ii) each zero $\xi \neq 0$ of $\left[J_{\nu}(x) / x^{ \pm \frac{1}{2}}\right]^{(4)}$ is transcendental and (iii) each zero $\xi \neq 0$ of $\left[J_{\nu}(x) / x^{ \pm \nu}\right]^{(4)}$ is transcendental except when $\nu=0$ with $\xi^{2}=3$.

PROOF. When $n=4$, and with $\lambda=\mu^{2}-\nu^{2}$, the differential equation (3) has as respective coefficients of $x y^{\prime}$ and $y$

$$
p_{4}(x)=-2(2 \mu+1) x^{2}-2(2 \mu+3) \lambda+2(\mu+1)(2 \mu+1)(2 \mu+3)
$$

and

$$
q_{4}(x)=-x^{4}+[(2 \mu+1)(2 \mu+3)-2 \lambda] x^{2}+\left(4 \mu^{2}+12 \mu+11\right) \lambda-\lambda^{2} .
$$

These expressions are calculated by applying (4) with $n=3$ to (8).

The algebraic zeros of $\mathcal{J}_{\nu, \mu}^{(4)}(x)$ arise from the common zeros of $p_{4}(x)$ and $q_{4}(x), \quad x \neq 0$.

When $\mu=-\frac{1}{2}, \quad p_{4}(x)$ can be zero only when $\lambda=0$ so that in this case $q_{4}(x)=-x^{4}$, which vanishes only when $x=0$. Thus, when $\mu=-\frac{1}{2}, \mathcal{J}_{\nu, \mu}^{(4)}(x)$ has no algebraic zeros other than perhaps $x=0$. Henceforth, we consider $\mu \neq-\frac{1}{2}$. To determine the common zeros of $p_{4}(x)$ and $q_{4}(x), \quad \mu \neq-\frac{1}{2}, \quad x \neq 0$, the value of $x^{2}$ for which $p_{4}(x)=0$ is substituted in $q_{4}(x)$. On simplification, $q_{4}(x)=0$ then becomes

$$
4 \lambda^{2}-2(2 \mu+1)\left(4 \mu^{2}+12 \mu+7\right) \lambda-\mu(\mu+1)(2 \mu+1)^{2}(2 \mu+3)^{2}=0 .
$$


Since $\lambda=\mu^{2}-\nu^{2}$. the solutions of the quadratic equation (12) yield (9). The values (10) result from replacing in the equation $p_{4}(x)=0$ the expression for $\nu^{2}$ given by (9). The restriction (11) merely restates the hypothesis that $\nu$ is rational. This proves the theorem. The assertions in the corollary are easy consequences in light of (12).

Remark. Condition (11), like the corresponding restriction in Theorem 3 implies the nonexistence of algebraic zeros in many cases. For example, if $\mu=1.2,3$, these requirements are not met and so neither $\mathcal{J}_{\nu, \mu}^{\prime \prime \prime}(x)$ nor $\mathcal{J}_{\nu, \mu}^{(4)}(x)$ have algebraic zeros when $\mu=1,2,3, x \neq 0$.

\section{RESULTANTS AND MAPLE V.}

Even in the special case $\mu=0$, the polynomials $p_{n}, q_{n}$ become substantially more complicated as $n$ increases. For example:

$$
p_{5}(x)=-x^{4}+\left(2 \nu^{2}+7\right) x^{2}-\nu^{4}-35 \nu^{2}-24,
$$

and

$$
\begin{gathered}
q_{5}(x)=2\left[x^{4}-6\left(\nu^{2}+1\right) x^{2}+5 \nu^{2}\left(\nu^{2}+5\right)\right], \\
p_{6}(x)=3\left[x^{4}-\left(6 \nu^{2}+11\right) x^{2}+5 \nu^{4}+75 \nu^{2}+40\right],
\end{gathered}
$$

and

$$
\begin{gathered}
q_{6}(x)=x^{6}-3\left(\nu^{2}+3\right) x^{4}+3\left(\nu^{4}+26 \nu^{2}+20\right) x^{2}-\nu^{6}-85 \nu^{4}-274 \nu^{4}, \\
p_{7}(x)=x^{6}-3\left(\nu^{2}+5\right) x^{4}+3\left(\nu^{4}+50 \nu^{2}+64\right) x^{2}-\nu^{6}-175 \nu^{4}-1624 \nu^{2}-720,
\end{gathered}
$$

and

$$
q_{7}(x)=3\left[-x^{6}+\left(9 \nu^{2}+17 x^{4}\right)-\left(15 \nu^{4}+190 \nu^{2}+120\right) x^{2}+7 \nu^{6}+245 \nu^{4}+588 \nu^{2}\right] .
$$

The method of $\S 1$ is, in principle, still applicable. In the case $n=8$, for example, we find that $p_{8}(x)$ and $q_{8}(x)$ have a common zero if, and only if,

$$
\begin{gathered}
-31907119104 \nu^{30}-847042707456 \nu^{28} \\
-4180678680576 \nu^{26}-614494258827264 \nu^{24} \\
-4099235438453760 \nu^{22}-16797585600728640 \nu^{20} \\
-44622481560865120 \nu^{18}-74605419864103690 \nu^{16} \\
-52227966835676760 \nu^{14}+89924792224537360 \nu^{12} \\
+340011463648979674 \nu^{10}+547044103885835061 \nu^{8} \\
+549201731824654896 \nu^{6}+359020825792238369 \nu^{4} \\
+144928821192067000 \nu^{2}+3040143522850000=0 .
\end{gathered}
$$

This equation has precisely one positive root. It is located between 1.25 and 1.3, the polynomial being positive when $\nu=1.25$ and negative when $\nu=1.3$. For the root to be rational (expressed in lowest terms), its numerator must be a factor of the constant term

$$
3040143522850000=2^{4}\left(3^{5}\right)\left(7^{5}\right)\left(53^{3}\right),
$$

its denominator a factor of the leading coefficient

$$
31907119104=2^{20}\left(3^{2}\right)\left(7^{2}\right)(23) .
$$

One can show by checking that no such rational root is possible, so $J_{\nu}^{(8)}(x)$ has no algebraic zero, except possibly $x=0$, for $\nu$ rational.

Rather than giving more and more complex ad hoc arguments for larger values of $n$, we will use the concept of resultant. The resultant (or eliminant) [4] of two polynomials $p$ and $q$ is a 
function $r$ of the coefficients which vanishes if and only if the polynomials have a common root. The resultant may be expressed as a determinant involving the coefficients or it may be found [6] using the Euclidean (greatest common divisor) algorithm. The proofs of Theorems 3, 4 and 5 may be rearranged by finding the values of $\mu$ and $\nu$ for which resultant $r_{n}(\mu, \nu)$ of $p_{n}$ and $q_{n}$ vanishes. We then substitute these values back into $p_{n}$ and $q_{n}$ and check to see whether they have a common non-zero root, i.e., whether their g.c.d. is anything other than a power of $x$.

For example, in Theorem 3, the resultant of $p_{2}(x)$ and $q_{2}(x)$ is $2 \mu+1$ which vanishes for $\mu=-1 / 2$. For $\mu=-1 / 2$, we get $p_{2}(x)=0$ and $q_{2}(x)=x^{2}-\nu^{2}+1 / 4$ which have a common non-zero root only if $\nu^{2} \neq \frac{1}{4}$.

For $n \geq 5$, we concentrate on the special cases $\mu=0,1 / 2, \nu$ and $-1 / 2$.

2.1 The case $\mu=0$. We used Maple V (Release 1) to form the polynomials $p_{n}$ and $q_{n}$, and to set up [1, pp.178-179] and factor their resultant $r_{n}$, a polynomial in $\nu$. Here is the procedure used on a SUN 4/75 at York University.

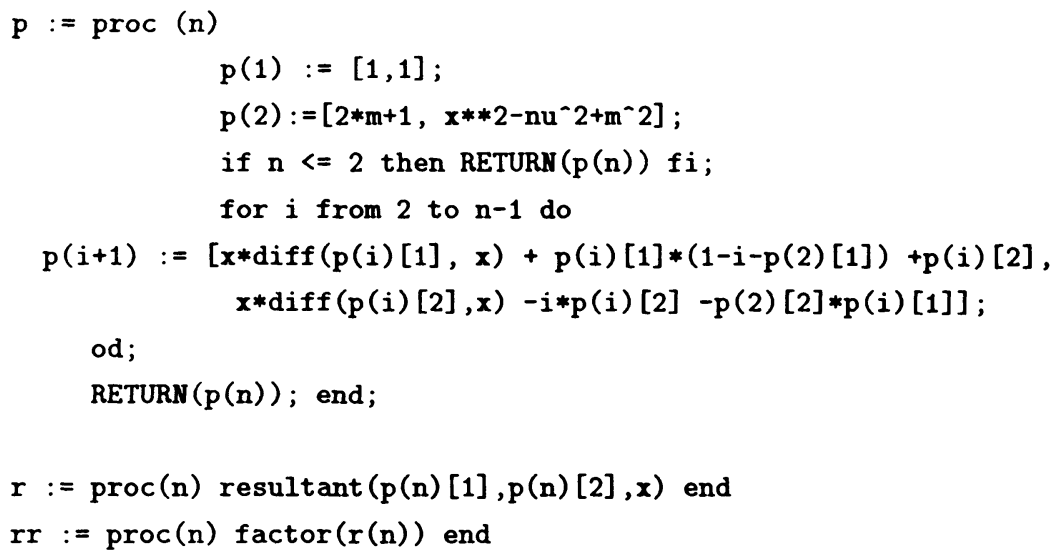

The following expressions were obtained for the first eight resultants factored over the rationals.

$$
\begin{gathered}
r_{1}(\nu)=1, r_{2}(\nu)=1, \\
r_{3}(\nu)=4(\nu-1)^{2}(\nu+1)^{2}, \\
r_{4}(\nu)=64 \nu^{4}\left(7+2 \nu^{2}\right)^{2} \\
r_{5}(\nu)=16\left(877 \nu^{2}+493 \nu^{4}+448 \nu^{6}+432\right)^{2}, \\
r_{6}(\nu)=11664\left(-10000-16723 \nu^{2}-48749 \nu^{4}\right. \\
\left.-47208 \nu^{6}-12576 \nu^{8}+256 \nu^{10}\right)^{2} \\
r_{7}(\nu)=746496\left(10800 \nu^{14}+1045744 \nu^{12}-3311544 \nu^{10}-51138608 \nu^{8}\right. \\
\left.-103853517 \nu^{6}-105987611 \nu^{4}-48750264 \nu^{2}-12150000\right)^{2} \\
r_{8}(\nu)=47775744\left(18464768 \nu^{18}+351700992 \nu^{16}+20996299776 \nu^{14}\right. \\
+185371620544 \nu^{12}+278668777744 \nu^{10}-72563241789 \nu^{8} \\
\left.-747805126038 \nu^{6}-1012303674997 \nu^{4}-626716971000 \nu^{2}-204205050000\right)^{2}
\end{gathered}
$$


Hence $\nu= \pm 1$ are the only (rational) roots of $r_{3}(\nu)$. This is consistent with part (i) of the Corollary to Theorem 4. We carried out the computation as far as $n=18$ and there are no further rational roots of $r_{n}(\nu)$. Hence, for these values of $n$, there is no possibility of a common root of $p_{n}$ and $q_{n}$ and hence no non-zero algebracc root of $J_{\nu}^{(n)}(x)$, when $\nu$ is rational. Our results for $\mu=0$ can be summarized as follows:

THEOREM 6. If $\nu$ is rational and $x \neq 0$, then all zeros of $J_{\nu}^{(n)}(x), n=0,1,2, \ldots, 18$ are transcendental'with the exception of the zeros $\pm \sqrt{3}$ which occur in the cases $n=3, \nu= \pm 1$ and $n=4, \nu=0$.

2.2. The case $\mu=1 / 2$. We used Maple $V$ again to set up the polynomials $p_{n}$ and $q_{n}$ and their resultant $r_{n}$, factored over the rationals. The first few results are as follows:

$$
\begin{gathered}
r_{1}(\nu)=1, r_{2}(\nu)=4, \\
r_{3}(\nu)=\frac{1}{4}(2 \nu-5)^{2}(2 \nu+5)^{2}, \\
r_{4}(\nu)=\left(216 \nu^{2}-247+16 \nu^{4}\right)^{2}, \\
r_{5}(\nu)=4\left(36324 \nu^{2}+9909+3056 \nu^{4}+1216 \nu^{6}\right)^{2}, \\
r_{6}(\nu)=(729 / 4)\left(8 \nu^{2}+13\right)^{2}\left(256 \nu^{8}-29952 \nu^{6}-155808 \nu^{4}-16848 \nu^{2}-845743\right)^{2}, \\
r_{7}(\nu)=(729 / 1024)\left(11059200 \nu^{14}+2105987072 \nu^{12}-31126270976 \nu^{10}-410547792640 \nu^{8}\right. \\
\left.-1878589556672 \nu^{6}-3941745616400 \nu^{4}-3183516258012 \nu^{2}-3944054162017\right)^{2}, \\
r_{8}(\nu)=729\left(5754585088 \nu^{18}+146218352640 \nu^{16}+24293948915712 \nu^{14}\right. \\
+179297378451456 \nu^{12}-628056658616320 \nu^{10}-8129678421220864 \nu^{8} \\
-31107275263623680 \nu^{6}-71858687471992512 \nu^{4}-82804731870624480 \nu^{2} \\
-55560127536055125)^{2}
\end{gathered}
$$

The calculations were carried as far as $n=18$. The only case where a rational root exists is the case $n=3, \nu^{2}=25 / 4$; the corresponding roots of $\left[x^{-1 / 2} J_{\nu}(x)\right]^{(3)}$ are at $\pm \sqrt{12}$. This agrees with part (ii) of the Corollary to Theorem 4.

We summarize our results as follows:

THEOREM 7. If $\nu$ is rational and $x \neq 0$, then all zeros of $\left[x^{-1 / 2} J_{\nu}(x)\right]^{(n)}, n=$ $0,1, \ldots, 18$ are transcendental with the exception of the roots $\pm \sqrt{12}$ occurring for $n=3, \nu^{2}=$ $25 / 4$.

2.3. The case $\mu=\nu$. In contrast to the situation encountered in Theorems 6 and 7 , here the functions $r_{n}(\nu)$ appear to have rational roots for every $n \geq 2$. So we have to check further to see if these rational roots correspond to non-zero common roots of $p_{n}(x)$ and $q_{n}(x)$. We again have recourse to Maple $\mathrm{V}$, first to evaluate the roots of $r_{n}(\nu)$, then to find the greatest common divisor (using ged [1, pp. 100-101]) of the polynomials $p_{n}(x)$ and $q_{n}(x)$ in the special cases when these this roots are substituted for $\nu$. The Maple procedure of $\S 2.1$ was augmented as follows: 


\begin{tabular}{|c|c|c|}
\hline$n$ & $\nu$ root & gcd \\
\hline \hline 2 & $-1 / 2$ & $x^{2}$ \\
\hline 3 & $-1 / 2$ & $x^{2}$ \\
& -1 & $x^{2}$ \\
\hline 4 & 0 & $x^{2}-3$ \\
& $-1 / 2$ & $x^{4}$ \\
& -1 & $x^{2}$ \\
& $-3 / 2$ & $x^{2}$ \\
\hline 5 & 1 & $x^{2}-15$ \\
& $-1 / 2$ & $x^{4}$ \\
& -1 & $x^{2}$ \\
& $-3 / 2$ & $x^{2}$ \\
& -2 & $x^{2}$ \\
\hline 6 & $-1 / 2$ & $x^{6}$ \\
& -1 & $x^{2}$ \\
& $-3 / 2$ & $x^{4}$ \\
& -2 & $x^{2}$ \\
& $-5 / 2$ & $x^{2}$ \\
\hline 7 & $-1 / 2$ & $x^{6}$ \\
& -1 & $x^{2}$ \\
& $-3 / 2$ & $x^{4}$ \\
& -2 & $x^{4}$ \\
& $-5 / 2$ & $x^{2}$ \\
& -3 & $x^{2}$ \\
\hline
\end{tabular}

\begin{tabular}{|c|c|c|}
\hline$n$ & $\nu$ root & gcd \\
\hline \hline 8 & $-1 / 2$ & $x^{8}$ \\
& -1 & $x^{2}$ \\
& $-3 / 2$ & $x^{6}$ \\
& -2 & $x^{4}$ \\
& $-5 / 2$ & $x^{4}$ \\
& -3 & $x^{2}$ \\
& $-7 / 2$ & $x^{2}$ \\
\hline 9 & $-1 / 2$ & $x^{8}$ \\
& -1 & $x^{2}$ \\
& $-3 / 2$ & $x^{6}$ \\
& -2 & $x^{4}$ \\
& $-5 / 2$ & $x^{4}$ \\
& -3 & $x^{4}$ \\
& $-7 / 2$ & $x^{2}$ \\
& -4 & $x^{2}$ \\
\hline 10 & $-1 / 2$ & $x^{10}$ \\
& -1 & $x^{2}$ \\
& $-3 / 2$ & $x^{8}$ \\
& -2 & $x^{4}$ \\
& $-5 / 2$ & $x^{6}$ \\
& -3 & $x^{4}$ \\
& $-7 / 2$ & $x^{4}$ \\
& -4 & $x^{2}$ \\
& $-9 / 2$ & $x^{2}$ \\
\hline
\end{tabular}

\begin{tabular}{|c|c|c|}
\hline$n$ & $\nu$ root & gcd \\
\hline \hline 11 & $-1 / 2$ & $x^{10}$ \\
& -1 & $x^{2}$ \\
& $-3 / 2$ & $x^{8}$ \\
& -2 & $x^{4}$ \\
& $-5 / 2$ & $x^{6}$ \\
& -3 & $x^{6}$ \\
& $-7 / 2$ & $x^{4}$ \\
& -4 & $x^{4}$ \\
& $-9 / 2$ & $x^{2}$ \\
& -5 & $x^{2}$ \\
\hline 12 & $-1 / 2$ & $x^{12}$ \\
& -1 & $x^{2}$ \\
& $-3 / 2$ & $x^{10}$ \\
& -2 & $x^{4}$ \\
& $-5 / 2$ & $x^{8}$ \\
& -3 & $x^{6}$ \\
& $-7 / 2$ & $x^{6}$ \\
& -4 & $x^{4}$ \\
& $-9 / 2$ & $x^{4}$ \\
& -5 & $x^{2}$ \\
& $-11 / 2$ & $x^{2}$ \\
\hline
\end{tabular}

Table 1: Case $\mu=\nu$

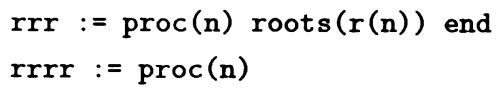

Table 1 gives the output of this procedure in the case $\mu=\nu$.

The left column contains values of $n$. The second contains the rational values of $\nu$ for which the resultant of $p_{n}$ and $q_{n}$ vanish and the third column contains the corresponding gcd's for $p_{n}$ and $q_{n}$. The only gcd's with a root other than 0 in the table correspond to the cases $n=4, \nu=0$ and $x^{2}=3$ (see part (i) of the Corollary to Theorem 5) and $n=5, \nu=1$ and $x^{2}=15$. We have carried the table to $n=13$ without encountering any other exceptional cases. This proves the following:

THEOREM 8. If $\nu$ is rational and $x \neq 0$, then all zeros of $\left[x^{-\nu} J_{\nu}(x)\right]^{(n)}, n=1, \ldots, 13$ are transcendental except for zeros at $\pm \sqrt{3}$ in case $n=4$ and $\nu=0$ and at $\pm \sqrt{15}$ in case $n=5$ and $\nu=1$.

The Table and its continuation suggest a number of conjectures: 
(a) That $r_{n}(\nu)$ is a polynomial of degree $n(n-1)$ in $\nu$.

(b) That $r_{n}(\nu)$ has exactly $n-1$ rational roots in case $n \neq 4,5$ and $n$ rational roots in case $n=4.5$.

(c) That the rational roots of $r_{n}(\nu)$ are $-m / 2, m=1, \ldots, n-1$ plus the additional root 0 in case $n=4$ and 1 in case $n=5$.

2.4. The case $\mu=-1 / 2$. In this case, we get

$$
\begin{gathered}
r_{2}(\nu)=0, \\
r_{3}(\nu)=\frac{1}{4}(2 \nu-1)^{2}(2 \nu+1)^{2}, \\
r_{4}(\nu)=(2 \nu-1)^{4}(2 \nu+1)^{4}, \\
r_{5}(\nu)=324(2 \nu-1)^{4}(2 \nu+1)^{4}\left(4 \nu^{2}+1\right)^{2}, \\
r_{6}(\nu)=729(2 \nu-1)^{6}(2 \nu+1)^{6}\left(-224 \nu^{2}+16 \nu^{4}-457\right)^{2}, \\
r_{7}(\nu)=(18225 / 1024)(2 \nu-1)^{6}(2 \nu+1)^{6}\left(20 \nu^{2}-29\right)^{2}\left(432 \nu^{4}+16200 \nu^{2}-7277\right)^{2}\left(4 \nu^{2}+17\right)^{2}, \\
r_{8}(\nu)=59049(2 \nu-1)^{8}(2 \nu+1)^{8}\left(2606460448 \nu^{4}+4463010256 \nu^{2}+4314561883\right. \\
\left.346449152 \nu^{6}+22256384 \nu^{8}+1605632 \nu^{10}\right)^{2} .
\end{gathered}
$$

What appears to be happening here is that, for each $n \geq 3$, the polynomial $r_{n}(\nu)$ has exactly two rational zeros $\frac{1}{2}$ and $-\frac{1}{2}$, but these never give rise to common zeros of $p_{n}$ and $q_{n}$. This has been verified for $n=3, \ldots, 15$ using a procedure similar to that used in the proof of Theorem 8. Taking into account the special case arising in Theorem 3, we have:

THEOREM 9. If $\nu$ is rational and $x \neq 0$, then all zeros of $\left[x^{1 / 2} J_{\nu}(x)\right]^{(n)}, n=1, \ldots, 15$, are transcendental, except for zeros at $\pm \sqrt{\nu^{2}-1 / 4}$ in case $n=2$ and $\nu^{2} \neq \frac{1}{4}$.

\section{CLOSING REMARKS.}

The results reported here suggest the following two questions (for which we expect negative answers):

1. Is there any pair of values $\nu, n$, where $\nu$ is rational and $n=19,20, \ldots$, such that $J_{\nu}^{(n)}(x)$ has an algebraic zero other than $x=0$ ?

2. Is there any pair of values $\nu, n$, where $\nu$ is rational and $n=19,20, \ldots$, for which there is more than one algebraic $\xi \neq 0$ such that $J_{\nu}^{(n)}( \pm \xi)=0$ ?

Theorems 7, 8 and 9 suggest analogous questions and conjectures for the zeros of $\left[x^{1 / 2} J_{\nu}(x)\right]^{(n)}$, $\left[x^{-\nu} J_{\nu}(x)\right]^{(n)}$ and $\left[x^{-1 / 2} J_{\nu}(x)\right]^{(n)}$, respectively.

The degrees of the polynomials $p_{n}, q_{n}$ corresponding to $\mathcal{J}_{\nu, \mu}(x), \mu \neq-\frac{1}{2}$, can be determined precisely: They are $\operatorname{deg}\left(p_{n}\right)=2[(n-1) / 2]$ and $\operatorname{deg}\left(q_{n}\right)=2[n / 2]$, where [.] denotes the greatest integer function. These values provide upper bounds for the numbers of possible algebraic zeros of $\mathcal{J}_{\nu, \mu}^{(n)}(x)$, but presumably quite wide of the mark.

ACKNOWLEDGMENTS. The authors' research was supported by the Natural Sciences and Engineering Research Council of Canada. We are indebted to Jason Brown of York University 
and Anders Källström of Uppsala University for performing symbolic algebraic calculations for an earlicr version of this paper. The success of these efforts led us to the more systematic use of Maple reported in $\S 2$.

\section{REFERENCES}

1. CHAR, B. W, ET AL., Maple V Library Reference Manual, Springer-Verlag, New York, 1991.

2. SHIDLOVSKII, A. B., Transcendental Numbers, Translated from the Russian by N. Koblitz, Walter de Gruyter, Berlin/New York, 1989.

3. SIEGEL, C.L., Über einige Anwendungen diophantischer Approximationen, Abh. Preussischer Akad. Wiss. Phys-Math. Kl. 1929, Nr. 1 (= Gesammelte Abhandlungen, vol. 1, 209-266, Springer. Berlin/ Heidelberg/New York, 1966).

4. VAN DER WAERDEN, B. L., Modern Algebra, vol. 1, revised English ed., Ungar, New York, 1953.

5. WATSON, G. N., A Treatise on the Theory of Bessel Functions, 2nd ed. Cambridge University Press. Cambridge/London/New York, 1944.

6. WEBER, H., Lehrbuch der Algebra, 1. Band, 2nd ed., 1898; reprinted, Chelsea, New York, 1961. 


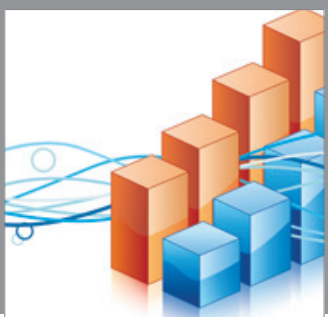

Advances in

Operations Research

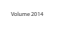

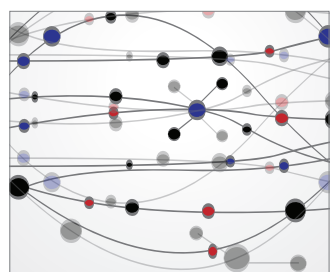

\section{The Scientific} World Journal
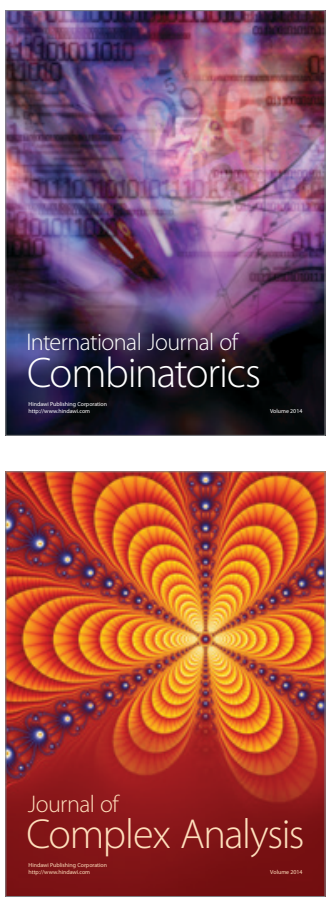

International Journal of

Mathematics and

Mathematical

Sciences
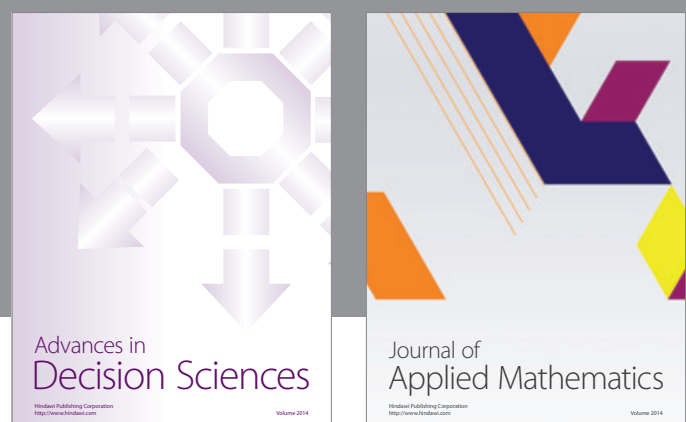

Journal of

Applied Mathematics
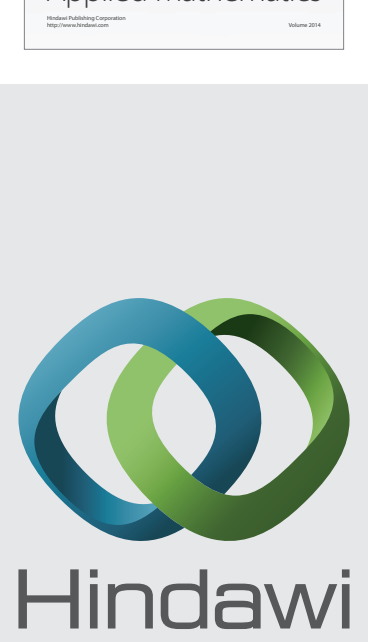

Submit your manuscripts at http://www.hindawi.com
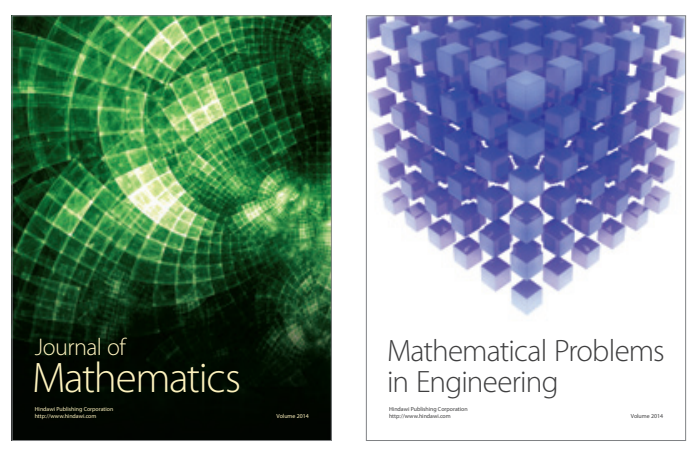

Mathematical Problems in Engineering
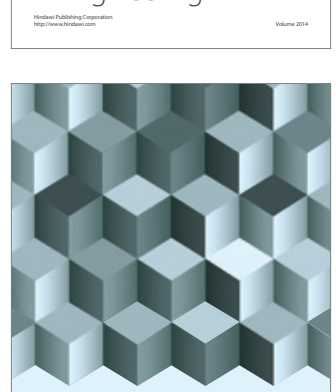

Journal of

Function Spaces
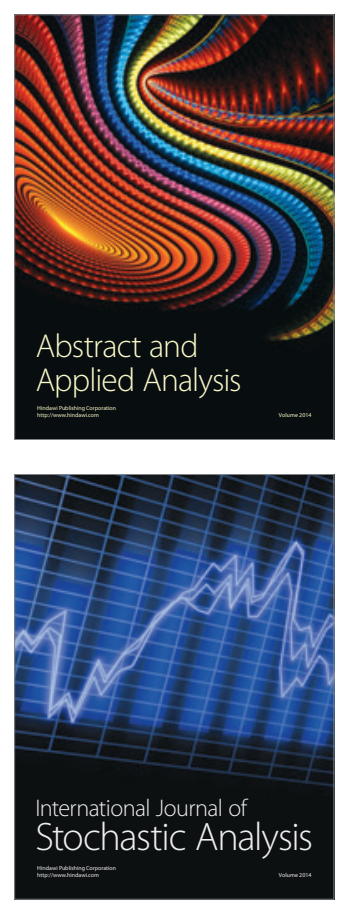

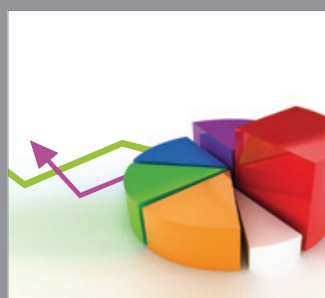

ournal of

Probability and Statistics

Promensencen
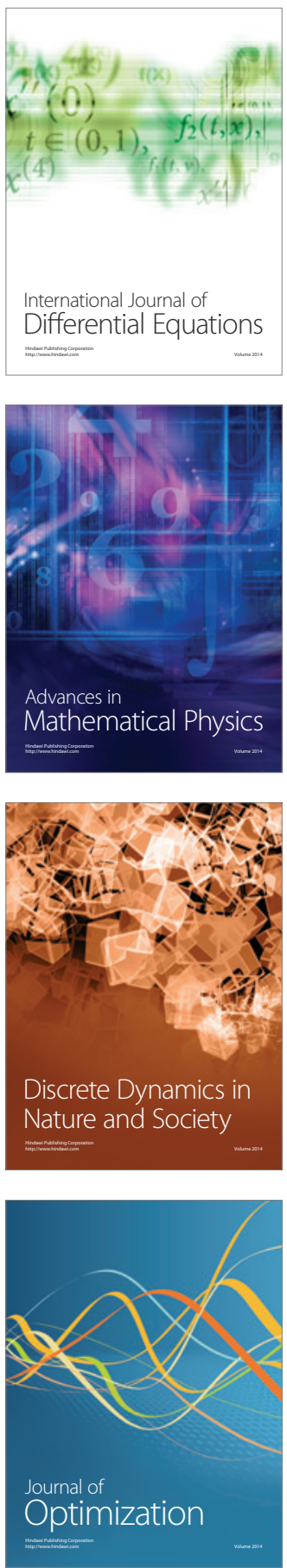